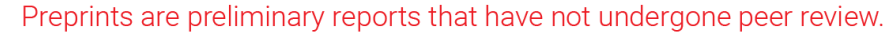 They should not be considered conclusive, used to inform clinical practice, or referenced by the media as validated information. \\ Epidemiology of Sheep Lice and Efficacy Evaluation of $60 \%$ Diazinon and $1 \%$ Ivermectin against Bovicola ovis in Sayint District, South Wollo, Ethiopia
}

\section{Simegn Legesse}

Woldia University

Mussie Hailemelekot

Bahir Dar University College of Agriculture and Environmental Sciences

Habtamu Tamrat ( $\square$ habtamut1978@gmail.com )

Bahir Dar University College of Agriculture and Environmental Sciences

Yeshwas Ferede

Bahir Dar University College of Agriculture and Environmental Sciences

Research article

Keywords: Bovicola ovis, Efficacy, Ethiopia, prevalence, Sheep, Sayint District

Posted Date: October 23rd, 2020

DOI: https://doi.org/10.21203/rs.3.rs-72766/v1

License: (a) (i) This work is licensed under a Creative Commons Attribution 4.0 International License.

Read Full License 


\section{Abstract \\ Background}

Sheep lice, caused by Bovicola ovis are very common in Ethiopia. It can result decreased production and reproduction, downgrading and rejection of skins in tannery industries. A cross-sectional and experimental study were conducted in Sayint District, South Wollo, Ethiopia which aims to determine the prevalence of sheep lice with the associated risk factors, identify the major sheep lice species and evaluate the efficacy of $60 \%$ Diazinon and $1 \%$ Ivermectin against Bovicola ovis. Study kebeles and animals were selected using purposive and multistage sampling techniques. Accordingly, a total of 232 sheep were sampled to estimate the prevalence of sheep lice. Fifteen sheep for in-vivo and 80 Bovicola ovis lice for in-vitro test were used for drug efficacy trial, by using completely randomized design.

\section{Results}

The overall prevalence of sheep lice in this study was $48 \%$. Hair length $(O R=2, P=0.00)$, body condition $(O R=1.9, P=0.02)$, agro ecology $(O R=1.19, P=0.00)$ and season $(O R=2, P=0.01)$ were significantly $(P$ $<0.05)$ associated with sheep lice infestation. The dominant sheep lice species in the study area were Bovicola ovis with prevalence of $83 \%$ and mixed infestation of $17 \%$ Bovicola ovis with Linognatus ovillus. In this study, the efficacy of $60 \%$ Diazinon and $1 \%$ Ivermectin against Bovicola ovis were $97 \%$ and $81 \%$, respectively.

\section{Conclusion}

The overall prevalence of sheep lice infestation in the present study area was higher, in which Bovicola ovis was predominant and resistant against $1 \%$ Ivermectin. Therefore, tailor made intervention is required with a view to reducing the prevalence of sheep lice infestation and addressing drug resistance in the study area.

\section{Background}

Ectoparasite of sheep are very common and widely distributed in all agro-ecological zones in Ethiopia and sheep lice are considered as one of potential threats which pose a serious economic loss to the farmer, the tanning industry and the country as a whole [1,2]. Skin problems caused by lice can result in mortality, decreased production and reproduction, downgrading and rejection of skins [3]. Genus Linognatus are obligate hematophagous ectoparasites of mammals and these ectoparasites harm the health of their hosts by blood sucking and this leads primarily to enormous losses of blood. Furthermore, the biting sites become super infected with bacteria and these wounds attract licking flies $[4,5]$. Bovicola ovis is one of the most common lice found on sheep. They feed by chewing on the skin surface and surface debris and produce itching, irritation and possible hair loss. An allergic skin hypersensitivity 
reaction to lice is another cause for "Cockle" in processed sheep skins [6]. In this regard, lice infestation in sheep cause cockle and it is the commonly reported disease in Ethiopia [7-9].

Control of sheep ectoparasites is currently an integration of sheep husbandry, farm management and insecticide use. Chemical control remains the most important and widely used strategy against most insect pests around the world. However, studies have shown that multiple resistance mechanisms in insects confer resistance to a range of insecticide classes [10]. For instance, the result of widespread chemical use has been the sequential development of resistance of Bovicola ovis [11]. Usually, resistance will be suspected through lack of efficacy during clinical use. However, lack of efficacy could also occur due to inadequate application of a product which leads to resistant ectoparasite species against acaricides [12].

To this end, there is a paucity of information on the epidemiology of sheep lice; the risk factors associated lice infestation in the study area and insecticide efficacy in Amhara region in general and in Sayint district of south Wollo zone in particular. Therefore, this study was initiated to determine the prevalence of sheep lice in the study area, to investigate the major risk factors associated with sheep lice infestation in the district, to identify the existing sheep lice species in the study area, to evaluate the efficacy of $60 \%$ Diazinon and $1 \%$ Ivermectin against sheep lice.

\section{Methods}

\section{Study Area}

The study was conducted in Sayint district which is found in the western part of south Wollo zone, Amhara National Regional State which is located 590 km, $659 \mathrm{~km}$ and $189 \mathrm{~km}$ from Addis Ababa, Bahir Dar and Dessie, respectively. The altitude of the district ranges from 500 to 4247 m.a.s.l. The area constitutes three agro climatic zones, highland (>2500 m.a.s.I), midland (1500-2500 m.a.s.I.) and lowland ( $<1500$ m.a.s.l.), which comprises about $42.8 \%, 34.6 \%, 22.6 \%$ of the total area, respectively [13]. The average temperature of the district ranges from $4-40^{\circ} \mathrm{C}$ and annual rain fall ranges from $800 \mathrm{~mm}$ to 1000 $\mathrm{mm}$ [14]. The main rainy season in this area is between early June and the end of September, in addition a small rain occurs between early February and end of April [13]. Livestock rearing is the most important economic activity in which shoat and cattle are the major livestock species kept in the area. The total number of animals kept in the district were 141, 921 cattle, 222, 996 shoat, 23,982 equine, 143,872 poultry.

\section{Study Animals}

Local breed sheep and their crosses with Awassi of both sexes and all ages managed under extensive production system were the study animal.

\section{Study Design}




\section{Cross-sectional study}

A cross-sectional study was applied from October 2018 to April 2019 in order to estimate the prevalence of sheep lice and to identify the major lice species of sheep and determine associated risk factors in the district. Sex, age, hair length, body condition, housing management (whether the sheep is housed separately or with other animals; as separate and mixed), agro ecology (highland, midland and lowland) and season of sampling (wet and dry season) was recorded and documented along with the sample collection. Sheep were categorized into two age groups; young (< one year) and adult ( $>$ one year), as described by [15]. Age estimation was done by dentition as indicated in [16]. Body condition scores of sheep were determined as either poor or good according to [17].

\section{Sampling Method}

Preliminary data were sourced from the respective district of agricultural office to have lists of kebeles and list of sheep producing households in the district. Purposive sampling technique was employed to select the study district based on the availability of sheep. Two stage stratified sampling was applied and proportional samples from each kebele were drawn. Accordingly, 34 kebeles were stratified based on agro ecology (nine highlands, nine midland and sixteen lowland). Three kebeles were selected randomly representing the three agro ecologies. Hence, 102, 60 and 70 sheep were sampled from Yegodo, Yegoda and Ada, respectively. The selected kebeles were visited and sampled twice during the study period on October 2018 and January 2019 representing the wet and dry seasons, respectively.

\section{Sample Size estimation}

The sample size calculation used in this study was calculated according to [18], using an expected prevalence of $6.94 \%$ in Kutaber [19], a 95\% confidence interval and a required absolute precision of $5 \%$. In total 99 sheep were used for this study. However, the sample size was increased to 232 to enhance the precision.

\section{Experimental study}

Due to limited number of experimental unit, completely randomized design was used to evaluate the efficacy of $60 \%$ Diazinon and $1 \%$ Ivermectin against Bovicola ovis in local sheep. About 15 naturally Bovicola ovis infested (greater or equal to 100) local male sheep aged between two to four years that have moderate body condition with short and medium hair size and not recently treated with any acaricide were used for this study. Study animals were obtained from three willing local farmers. Before the commencement of the trial, study farmers were trained how to uniformly manage their animals by the investigator and separate houses for each group of sheep was prepared and cleaned. Lists of individual sheep identification number were made and lottery method of randomization was used to allocate into three groups having five sheep each, and followed for 28 days. After 15 days of acclimatization period, by proper straining and handling of animal, first group were sprayed with $60 \%$ Diazinon, the second group 
was given $1 \%$ Ivermectin injection subcutaneously as per manufacture's recommended dose and the third group was left untreated as a control. Both in-vivo and in-vitro efficacy trials were performed for Diazinon as per the protocols given by [20], while only in-vivo efficacy trial was conducted for Ivermectin as per [21]. Lice count was performed every 7 days for treatment and control groups and recorded starting from day 0 prior to treatment, days 7, 14, 21 and 28 according to [22]. 60\% Diazinon contains an active ingredient of $600 \mathrm{~g} / \mathrm{l}$ Diazinon produced by Adamitulu pesticide processing share company, Addis Ababa, Ethiopia.1\% Ivermectin was manufactured by Bash Pharmaceutical Company, Khartoum, Sudan, and it was imported by Rang veterinary company, Addis Ababa, Ethiopia.

In-vivo trial: Each selected sheep in treatment and control group was visually examined and lice from individual animal was counted and recorded separately for $60 \%$ Diazinon and $1 \%$ Ivermectin for the invivo evaluation. The total lice count was applied by direct examination of the body with naked eyes. Lice from each sheep was counted using parting method, classifying the body into five parts: neck, shoulder, withers, flank and rump after marking 4 partings per site on both sides of the body $[20,22]$. Thus, twenty sites on each side of the body were examined by parting the fleece about $10 \mathrm{~cm}$ and counting all live lices observed, so that , the total count from 40 sites constitutes the body count for each animal. The total lice count per animal was estimated by summation of the lice number at each site. Finally, lousicide activity was checked using arithmetic mean louse count for $60 \%$ Diazinon and $1 \%$ lvermectin group separately along with a control group which was calculated according to [23]. Thus, mortality, 98-100\% indicates susceptibility and less than $98 \%$ is suggestive of the existence of resistance [24].

In-vitro trial: sufficient live and motile lice were collected manually from naturally infested sheep that came into clinics and immediately taken into the laboratory. After species identification, 80 Bovicola ovis louse were randomly allocated into treatment and control groups. Each group contains 4 replicates having 10 lice placed on each petri dish as per the protocol provided by [25]. Then, $60 \%$ Diazinon was diluted in water according to the manufacturer's recommendation (1:1000) and lice in the treatment group were immersed completely in $0.5 \mathrm{ml}$ of this solution of $60 \%$ Diazinon for one min [26] and the control lice in the same amount of distilled water. After one minute, the solution soaked and dried using Whatman filter paper. Then, the vital signs in which lice exhibited after treatment was checked at 10, 30, 60, 120 minutes, as well as 6 and 12 hours of contact time under microscope [25].

Accordingly, recording of vital signs of lice in the treatment and control groups was held in each visiting time as described by [26]. For the calculation of mortality, highly stringent criteria was used and lice only judged as dead if they are in the categories 3 or 4 after 10, 30, 60 and 120 minutes as well as after 6 and 12 hours of contact time with $60 \%$ Diazinon[26]. The percentage mortality was calculated using a formula described by [27] as follows;

$$
\% \text { Mortality }=\frac{\text { Number of dead lice }}{\text { Total number of lice }} \times 100
$$


Where the insecticidal effect of Diazinon was classified as: "strong" when mortality is $>80 \%$, "moderate" mortality $80-60 \%$, "weak" mortality $60-40 \%$, "little or no activity" mortality $<40 \%$.

\section{Data Collection}

\section{Clinical examination}

Data were collected in two seasons (October and January) to observe the seasonal lice infestation variation between the late rainy and dry seasons of the area. After proper restraining, clinical examination of each sheep was done using multiple fleece partings in the opposite direction in which sheep's hair or wool normally rests. The skin was inspected carefully with naked eye and palpated across all parts of the sheep for the presence of ectoparasites and gross lesions that could be suggestive for clinical form of parasitic infestation [28]. Sheep having the parasite or lesions like alopecia and itching was considered as positive.

\section{Lice collection and identification}

Careful examination of the animal body, especially neck, shoulder, breast, ribs, back, and flank and rump areas was performed to detect the presence of lice by parting the body hair. Lice were collected manually and added into labelled universal bottles with $70 \%$ Ethanol. Collected samples were dispatched to Bahir Dar Animal Health Diagnostics and Investigation Center for laboratory analysis. Lice identification was done using stereo microscope according to the descriptions of [29].

\section{Data Management and Analysis}

Raw data obtained during sample collection and laboratory identification were checked, coded, and entered into Microsoft Excel spread sheet by the principal investigator and then the raw data was exported to STATA version 13 for analysis. Both univariable and multivariable logistic regression was used to examine and quantify the association between lice infestation and explanatory variables. These significant variables $(P<0.05)$ by univariable logistic regression were further evaluated by multivariable logistic regression to adjust the effect of confounding. Moreover, independent sample t-test was used to compare mean lice burden between treatment and control group

\section{Results}

\section{Prevalence of Sheep Lice}

The overall prevalence of sheep lice in the study area was $48.3 \%$ (112/232), with the highest prevalence of Bovicola ovis (83\%) followed by mixed infestation of Bovicola ovis with Linognatus ovillus (17\%). No sole infestation with Linognatus ovillus found by this study (Table 1 ).

Table 1. Overall and relative prevalence of sheep lice species in Sayint district 


\begin{tabular}{|lll|}
\hline & \multicolumn{1}{|c|}{$(\mathbf{N}=\mathbf{2 3 2})$} & \\
\hline Lice spp identified & \multicolumn{1}{c|}{ Number positive $(\mathrm{n})$} & \multicolumn{1}{c|}{ Relative Prevalence (\%) } \\
\hline Bovicola ovis & 93 & 83.04 \\
\hline Mixed infestation & 19 & 16.96 \\
\hline Total & 112 & 100 \\
\hline Overall & & $48.3^{\star}$ \\
\hline
\end{tabular}

*= total positive divided by total population $(\mathrm{N})$

\section{Risk factor analysis}

Five potential risk factors were significantly $(P<0.05)$ associated with sheep lice infestation using univariate logistic regression. These included hair length, housing management, body condition, agro ecology and season (Table 2). However, these potential risk factors were further evaluated by multivariable logistic regression.

Table 2. Univariable and multivariable logistic regression analyses of risk factors associated with sheep lice infestation in the study area 


\begin{tabular}{|c|c|c|c|c|c|c|c|}
\hline \multirow{3}{*}{ Variables } & \multirow{3}{*}{ Categories } & \multicolumn{4}{|c|}{$\begin{array}{l}\text { Univariable logistic regression Analyses } \\
\text { regression Analyses }\end{array}$} & \multicolumn{2}{|c|}{ Multivariable logistic } \\
\hline & & \multicolumn{6}{|c|}{ multivariate analyses } \\
\hline & & OR & $95 \% \mathrm{Cl}$ & $P$ value & OR & $95 \% \mathrm{Cl}$ & $P$ value \\
\hline \multirow[t]{2}{*}{ Sex } & Male & 0.9 & $0.56-1.58$ & 0.83 & & & \\
\hline & Female & & & & & & \\
\hline \multirow[t]{2}{*}{ Age } & Young & 1.4 & $0.85-2.41$ & 0.17 & & & \\
\hline & Adult & & & & & & \\
\hline \multirow[t]{2}{*}{ Hair length } & Short & 2.3 & $1.41-4.06$ & 0.00 & 2.18 & $1.2-3.9$ & 0.00 \\
\hline & Medium & & & & & & \\
\hline \multirow[t]{2}{*}{ House Mg't } & Separate & 0.4 & $0.24-0.76$ & 0.00 & & & \\
\hline & Mixed & & & & & & \\
\hline \multirow{2}{*}{$\begin{array}{l}\text { Body } \\
\text { condition }\end{array}$} & Poor & 2.5 & $1.4-4.3$ & 0.00 & 1.9 & $1.1-3.5$ & 0.02 \\
\hline & Good & & & & & & \\
\hline \multirow{3}{*}{$\begin{array}{l}\text { Agro } \\
\text { ecology }\end{array}$} & Highland & 4.0 & $2.1-7.6$ & 0.00 & 3.4 & $1.7-6.7$ & 0.00 \\
\hline & Midland & 5.0 & $2.5-10$ & 0.00 & 4.4 & $2.1-9.1$ & 0.00 \\
\hline & Lowland & & & & & & \\
\hline \multirow[t]{2}{*}{ Season } & Wet & 1.8 & $1.0-3.0$ & 0.02 & 2.0 & $1.1-3.6$ & 0.01 \\
\hline & Dry & & & & & & \\
\hline
\end{tabular}

Mg't=management

According to the final model, hair length, body condition, agro-ecology and season were significantly associated $(P<0.05)$ with sheep lice infestation (Table 2$)$. The odds of lice infestation in medium size hair coated sheep were 2.2 times higher as compared to sheep with short hair. Likewise, the odds of lice infestation was 1.9 times higher in poorly conditioned sheep as compared to well-conditioned ones. As compared to the lowland agro-climate, the odds of sheep lice infestation were 4.4 and 3.4 times higher in midland and highland agro-climates, respectively (Table 2). 
In-vitro efficacy test: based on the vital signs lice exhibit during the test, mortality was calculated. Hence, from lice treated with $60 \%$ Diazinon, 38 of them did not show any vital signs with 12 hours giving a mortality of $95 \%$. All lice in the control group had survived during the observation period. The test acaricide has killed $2.5 \%$ of lice at $10 \mathrm{~min}, 7.5 \%$ at $30 \mathrm{~min}$, and $10 \%$ at $60 \mathrm{~min}, 17.5 \%$ at $120 \mathrm{~min}, 82.5 \%$ at $360 \mathrm{~min}$ ( 6 hour) and $95 \%$ at $720 \mathrm{~min}(12$ hour). There was significant $(p=0.05)$ variation in percentage mortality of lice between $60 \%$ Diazinon treated and untreated group. The highest mortality was observed in the treatment group at 720 minutes (12 hour). The over all in vitro efficacy of $60 \%$ Diazinon was $95 \%$. The in-vitro post treatment mean number of dead lice per minute of time is shown in Fig 1 . The mean number of dead lice in the treated group had slightly increased from 30 to $120 \mathrm{~min}$ and consequently high mortality percentage was observed between 120 and 360 min, with the highest pick at 720 min Fig. 1. However, there was no any change in the untreated group throughout the study period.

In-vivo efficacy test: the overall in-vivo efficacy of Diazinon $60 \%$ was $99 \%$. Though very slight infestation on days 7 and 14 post treatment, there was a significant $(p<0.001)$ reduction in mean lice burden on treated sheep as compared to the untreated group. After the second round spray, on day 14, all sheep were found free of lice infestation while mean number of lice burden on untreated sheep was progressively increasing The mean number of lice burden reduction by $60 \%$ Diazinon among treated and untreated sheep before and after treatment is shown in Table 3. Before treatment, there was nearly similar mean lice burden on treated and untreated groups. Significant reduction of mean lice count was observed in treatment groups, while, a relatively high level of infestation was observed in untreated group Fig. $\mathbf{2}$.

Table 3.Mean lice count among treated and untreated group after application of $60 \%$ Diazinon

\begin{tabular}{|lllcll|}
\hline \multicolumn{7}{|c|}{ Mean number of lice count \pm SD } \\
\hline Treatment days & Treated, N=5 & Untreated, N= 5 & $\mathbf{t}$ & $\mathbf{d f}$ & P-value \\
\hline Day 0 & $446 \pm 283$ & $403 \pm 163$ & 0.29 & 6 & 0.086 \\
\hline Day 7 & $9 \pm 3$ & $414 \pm 317$ & -2.85 & 4 & 0.000 \\
\hline Day 14 & $15 \pm 10$ & $415 \pm 131$ & -6.80 & 4 & 0.055 \\
\hline Day 21 & $0.00 \pm 0.00$ & $465 \pm 178$ & -5.83 & 4 & 0.002 \\
\hline Day 28 & $0.00 \pm 0.00$ & $634 \pm 224$ & -6.31 & 4 & 0.001 \\
\hline
\end{tabular}

The in-vivo test showed that the efficacy of $1 \%$ Ivermectin was $81 \%$. Before the commencement of the treatment, there was nearly similar mean lice count among treated and untreated groups. Mean lice count reduction by $1 \%$ Ivermectin injection was observed from day 0 to day 14 , with a gradual increase from day 14 till the end of the experiment. A significant mean lice count reduction $(p=0.01)$ was observed among treated and untreated groups (Fig .3 Table 4).

Table 4. Mean lice burden on treated and untreated sheep after 1\% Ivermectin injection Sayint district 


\begin{tabular}{|lllccc|}
\hline \multicolumn{7}{|c|}{ Mean number of lice count $\pm \mathbf{S D}$} \\
\hline Treatment day & Treated, N=5 & Untreated, N= 5 & $\mathbf{t}$ & $\mathbf{d f}$ & P-value \\
\hline Day 0 & $373 \pm 33$ & $403 \pm 163$ & -.409 & 4.335 & .111 \\
\hline Day 7 & $90 \pm 39$ & $414 \pm 317$ & -2.275 & 4.120 & .000 \\
\hline Day 14 & $70 \pm 13$ & $415 \pm 131$ & -5.860 & 4.078 & .065 \\
\hline Day 21 & $79 \pm 8$ & $465 \pm 178$ & -4.837 & 4.015 & .003 \\
\hline Day 28 & $108 \pm 15$ & $634 \pm 224$ & -5.228 & 4.036 & .001 \\
\hline
\end{tabular}

\section{Discussion}

In the present study, the overall prevalence of sheep lice in the study area was $48.3 \%$ which is higher than other previous similar studies in Bale [30] (5.5\%), Gamo [31] (4.6\%), Afar region [32](11.6\%), eastern Ethiopia [33] (21.4\%), Tigray region [34] (1.3\%), Kombolcha [35](28.5\%) and in Bahir Dar [36](11.5\%) and [37](3.8\%). However, this report is found lower than [38] in Aba Jima district (83\%) and in Gondar [39] who reported (57\%). The possible reason for such high prevalence of lice in the study area includes poor management, feed scarcity and lack of quality veterinary services. The present result is consistent with [40] in Tiyo District (49\%), and [41] in Arsi Highland areas (54\%).

Bovicola ovis was the dominant lice species isolated by the present study $(83 \%)$ followed by mixed infestation of Bovicola ovis with Linognatus ovillus (17\%). This finding is consistent with the reports of [41] and [42] who reported $86 \%$ Bovicola ovis and $12 \%$ mixed infestation (Bovicola ovis with Linognatus ovillus) in Arsi. However, it is much higher than the reports of (28) and [42] who reported $29 \%$ and $73 \%$ Bovicola ovis, respectively. Similar report was also done in Wolmera district by [43] who reported $83.23 \%$ prevalence of Bovicola ovis in sheep.

In the present study There was a significant $(P<0.05)$ association between sheep lice infestation and hair length. Those sheep with medium hair coat are more likely to be infested as compared to short ones. This finding is in lined with other previous reports done by $[\mathbf{3 8}, 34]$.

According to the present study, poor conditioned sheep were more likely to be infested with sheep lice as compared to well-conditioned counterparts, which agrees with the results of other studies $[\mathbf{9}, \mathbf{3 6}, \mathbf{4 1}, \mathbf{4 4}$, 45] who reported poor body condition sheep were more affected by ectoparasite infestation than good body condition., Poor body condition can be exacerbated by itching and disturbance during grazing. This condition can compromise the nutritional status of sheep and leads to immune suppression. However, the present finding contradicts the findings of [38], who reported the prevalence of Bovicola ovis was higher in well-conditioned animals.

In the present study, sheep kept in midland $(\mathrm{OR}=4.4)$ and highland $(\mathrm{OR}=3.4)$ agro-climates were more likely to be infested by sheep lice as compared to lowland agro-climates. This finding is consistent with 
$[34,45]$, but it disagrees with $[2,44]$, who reported higher prevalence of lice was recorded in Highlands followed by midland and lowland areas. The reason for higher prevalence in midland and highland might be associated with the temperature and moisture requirement of Bovicola ovis as well as shearing practice of farmers in the study area. In midland areas, temperature and moisture are conducive for lice to multiply. In highland areas, as the climate is very cold, farmers don't practice wool shearing to protect their sheep from cold stress. This practice could predispose sheep to lice infestation.

A significant higher lice infestation rate was observed during dry season as compared to wet season. This finding is found compatible with other previous reports $[30,46]$, while it disagrees with others [31, 44], where higher prevalence of sheep lice was recorded in wet season than dry season. The significant discrepancy between dry and wet season prevalence could be associated with feed scarcity that can result in poor body condition. During dry period, feed scarcity is common which exposes sheep to starvation and poor body condition that lead to immune suppression.

The efficacy of Diazinon $60 \%$ against Bovicola ovis was $99 \%$ and $95 \%$ for in-vivo and in-vitro, respectively. Hence, as per [27] and [24], the drug is effective for the treatment of Bovicola ovis. This result is found in line with previous studies $[\mathbf{1 8}, \mathbf{4 7}]$, who reported the in-vitro and in-vivo efficacy of Diazinon was $100 \%$. However, it is found inconsistent with [48], who reported Diazinon was $82 \%$ effective.

The overall in-vivo efficacy of $1 \%$ Ivermectin was $81 \%$, which is below the standard for in-vivo trials set limit (98-100\%), suggestive of drug resistance. This result is far lower than the findings of [49], who reported that Ivermectin was $100 \%$ effective against sheep lice based on pour on formulations $[19,47$, 50]. The probable reason why reduced efficacy of $1 \%$ Ivermectin injection against Bovicola ovis could be associated with prolonged and inappropriate use of the drug, the frequent application of $1 \%$ Ivermectin drug with the same brand without rotation with other insecticide classes. The importance of these presumed factors associated with drug resistance was stated by [19]. It is also evident that lack of insecticide class rotation and high treatment frequency increases macro-cyclic lactones resistance of parasites [51, 52].

\section{Conclusion}

The present study revealed that there was a higher prevalence of sheep lice infestation in the study area, where Bovicola ovis was the predominant lice species affecting sheep. A number of potential risk factors were tested to determine significant association with sheep lice infestation. Hair length, body condition, agro ecology and season were significantly associated with lice infestation in sheep. The in-vivo and invitro trials showed that $60 \%$ Diazinon was more effective to treat Bovicola ovis, where as $1 \%$ Ivermectin fails to treat sheep infested with Bovicola ovis. Hence, subsequent application of tailor made intervention towards the identified risk factors along with rational choice of drugs against sheep lice is warranted to reduce the prevalence of sheep lice infestation in the study area.

\section{Abbreviations}


AGRIS International System for Agricultural Science and Technology

CDM Center for Disease Control and Prevention

CVMP Committee for Medicinal Products for Veterinary Use

OR Odd Ratio

m.a.s.I Meter above sea level

\section{Declarations}

\section{Ethics approval and consent to participate}

Experiment trial was done with the farmer's animal and oral consent was made with the owners after getting ethical clearance paper from the Board of School Research Ethics Review Committee.

\section{Availability of data and material}

Not applicable

\section{Consent of publication}

Not applicable

\section{Competing of interest}

The authors declare that they have no competing interests.

\section{Funding}

No funding was obtained for this study.

\section{Authors' contribution}

SL planning of the study, data collection and drafted the manuscript. MH participated in the planning of the study, revised the manuscript. HT participated in the planning of the study, analysed, interpreting and editing of the final manuscript. YF involve during planning of the study, interpreting the finding and editing of the final manuscript. All authors read and approved the final manuscript.

\section{Acknowledgements}

The authors would like to acknowledge Bahir Dar Animal Health Diagnostics and Investigation Center, Ethiopia for allowing there laboratory.

\section{References}


1. Worku B, Haileleul N, Sefinew A,Hailu M. Assessment on major factor that cause skin rejection at Mojo export tannery, Ethiopia. Trop Animal Health Production.2011; 43: 989-993

2. Bersissa $\mathrm{K}$, Kebede $\mathrm{B}$, Mesula Ectoparasites of sheep in three agro-ecological zones in central Oromia, Ethiopia. Journal of Veterinary Research.2012; 79: 1-7.

3. Tefera S, Abebe Effect of ectoparasite on quality of pickled skins and their impact on the tannery industry, in Amhara regional state. Ethiopian Journal of Small Ruminant Research.2007a; 69: 55-61.

4. William MS, Margo JP, Alan AK. Parasitic Diseases of Wild Mammals, $2^{\text {nd }}$ edition, lowa State University, Wiley-Blackwell.2001:255-263.

5. Heinz M, Khaled A, Fathy A, Sven K,Herbert P. Life cycle and attacks of ectoparasites on ruminants during the year in central Europe: Recommendations for treatment with insecticides. Parasitology research.2010; 107(2): 425-431.

6. Kufman PE, Koehler PG,Butler J External Parasites of Sheep and Goats. University of Florida. IFSA extension. 2012:168-200

7. Kassa B, Brook A, Yeshimebet C, Demeke T, and Solomon G. Improvement of sheep skin quality after treatment with diazinon against cockle. Ethiopian Veterinary Journal.2012;16(2):49-63

8. Yacob H. Ectoparasitism: Threat to Ethiopian small ruminant population and tanning industry. Journal of Veterinary Medicine and Animal Health. 2014;6: 25-33

9. Desalegn D, Minda A. Sheep Mange mites and Lice: Prevalence and risk Factors in Asella and its surroundings, South Eastern Ethiopia. Journal of Veterinary Science and Technology.2016;7:5

10. Dang K, Doggett SL, Singham GV, Lee CY. Insecticide resistance and resistance mechanisms in bed bugs, Cimex spp (Hemiptera: Cimicidae). Parasites and vectors.2017; 10 (1): 318.

11. Levot GW. Resistance and the control of lice on humans and production animals. International Journal for parasitology.2000; 30: 291-297.

12. Reflection paper on resistance in Ectoparasites. European Medicines Agency.2018:3-30

13. Tesfahun K. Vulnerability to food insecurity in three agro ecological zones in Sayint district, Ethiopia. A thesis submitted to university of Cape Town. 2009:41-46

14. Amhara Sayint woreda communication affairs. Short description about Amhara sayint woreda and its location. Retrieved from Amhara sayint woreda communication affairs .2018.

15. Gatenby MR. Sheep. In: Coste R and Smith JA (eds). The Tropical Agriculturalist. London: Macmillan and CTA (Wageningen).1991:6-11

16. Casburn G. How to tell the age of sheep. Prime fact $1481,2^{\text {nd }}$ NSW (New South Wales), Department of Primary Industries, WaggaWagga.2016:2

17. Russel A. Body condition scoring of sheep. In: E. Boden (Ed.) Sheep and Goat Practice. Bailliere Tindall, Philadelphia.1991:3.

18. Thrusfield M. Veterinary epidemiology. $3^{\text {rd }}$ edition. Blackwell science Ltd. Oxford, Great Britain. 2005; 133-194. 
19. Kindu W, Mohamed H, Wosen T, Dagnachew E. Determination of prevalence of external parasite on different species. Malaysian Orthopedic Journal of Proteomics and Bioinformatics.2018 7 ; (5): 263-269.

20. Sultan A, Abdu M,Mihreteab B. Evaluation of in vitro and in vivo Effectiveness of Diazinon against DamalinaActa Parasitologica Globalis.2014:5 (3): 198-205.

21. Worku A, Araya M, Ayalew D,Natnael M. Comparative efficacy of two different brands of Ivermectin against gastrointestinal Nematodes and ectoparasites of Sheep in Gondar Town, Northwest Ethiopia. Online Journal of Animal Feed Research.2018; 8 (2): 12-19.

22. Holdsworth PA, Vercruysse J, Rehbein S, Peter R, Letonja T,Green N. World for the advancement of veterinary parasitology (W.A.A.V.P.) for evaluating the efficacy of ectoparasiticides against biting lice and sheep ked on ruminants, Veterinary Parasitology. 2006; 136: 45-54.

23. Demonstration of efficacy of ectoparasiticides. CVMP Working Party on the Efficacy of Veterinary Medicines. Notes for Guidance. Commission of the European Communities, Brussels. Document No. III/3682/92EN. 1993

24. Guideline for Evaluating Insecticide Resistance in Vectors Using the CDC Bottle Bioassay, Center for Disease Control and Prevention. U.S department of Health and Human Services.2017:15-17.

25. Levot GW. Implications of insecticide resistance for the control of flystrike and lice on Sheep. Elizabeth Macarthur Agricultural Institute. NSW Agriculture, Camden. Proceedings of the FLICS Conference, Launceston. 2001

26. Heukelbach J, Speare R,Canyon D. Natural products and their application to control head lice: an evidence-based review. In: Brahma Chari G(ed) chemistry of natural products: recent trend and developments. Kerala, India. 2006a:1-26.

27. Askale G. In vitro efficacy of methanolic extracts of Vernoniaamygdalina, Croton macrostachyus, Ricinuscommunis and petroleum ether extract of Millettiaferruginea against Bovicolaovis and Rhipicephalus (Boophilus) decoloratus, MSc thesis, Addis Ababa University.2015:26-44

28. Yishak I, Tsegalem A, Befekadu U. Epidemiological study on ectoparasite infestation of small ruminants in Sodo Zuria District, Southern Ethiopia. Journal of Veterinary Medicine and Animal Health.2015;7 (4):140-144.

29. Wall R, Shearer D. Veterinary Ectoparasites: Biology, Pathology and Control. 2nd edition. Blackwell Science, UK. 2001:55-82.

30. Tesfaye B. Ectoparasite prevalence in small ruminant livestock of Ginir district in Bale Zone, Oromia Regional State, Ethiopia. Nessa .Journal of Veterinary Science. 2017; 1(1): 1-6.

31. Bereket $M$, Haba H, Sefinew A. Prevalence and risk factors associated to skin diseases in small ruminants in Gamo Gofa zone, south-Western Journal of Veterinary Medicine and Animal Health.2017; 9: 228-234.

32. Fikre Z, Hailegebriel B, Mu'uz G, Ahmed S, Ashenafi G. Epidemiology of major small ruminant ectoparasites and effectiveness of the control approaches employed in selected pastoral districts of 
Afar, Northeastern Ethiopia. Journal of Biology, Agriculture and Healthcare.2015;5(14): ISSN 22243208

33. Nateneal T, Tesfaheywet Z. Prevalence and Identification of Ectoparasites Fauna in Small Ruminants in Selected Areas of Eastern Ethiopia. African Journal of Basic and Applied Sciences.2015; 7 (5): 240-246.

34. Rahmeto A, Makelesh T, Bekele M, Desie S. Prevalence of Small Ruminant Ectoparasites and Associated Risk Factors in Selected Districts of Tigray Region, Ethiopia. Global Veterinarians.2011; 7(5):433-437.

35. Abebayehu T, Endris F, Berhanu M, Rahmeto A, Solomon M ,Endrias Z. Study on the prevalence of ectoparasite infestation of ruminanats in and around Kombolcha and damage to fresh goat pelts and wet blue (pickled) skin at Kombolch Tannary, Northestern Ethiopia. Ethiopian Veterinary Journal. 2011; 15 (2): 87-101.

36. Asmare A, Assefa K, Tewodrose F. Occurrence of small ruminant ectoparasites in and around Bahir Dar, Northwest Ethiopia. Advances in Biological Research.20126 (5): 170-176.

37. Dawit T, Mulugeta A, Tilaye D, Mengistie T. Ectoparasites of small ruminants presented at Bahir Dar Veterinary Clinic, Northwest Ethiopia. African Journal of Agricultural Research.2012;7(33): 46694674.

38. Hailegebriel B, Getachew T , Yacob H. Current status of ectoparasites in Sheep and management practices against the problem in ectoparasites controlled and uncontrolled areas of Arsi Zone in Oromia Region, Journal of Veterinary Science and Technology.2015; S10: 002.

39. Tewodrose F, Fasil W, Mersha C ,Malede B. Prevalence of Ectoparasites on Small Ruminants in and Around Gondar Town American-Eurasian Journal of Scientific Research.2012;7 (3): 106-111.

40. Zera A. A cross-sectional study on the prevalence of ectoparasite of sheep and the effectiveness of control in Tiyo and Diksis districts. Global Journal of Veterinary Medicine and Research.2017:5 (9): 174-180.

41. Eyob E, Diriba L, Birhanu A, Hani S. Prevalence and associated risk factors to lice infestation in sheep of arsi high land, Oromia regional state, Ethiopia. Journal of Veterinary Science and Technology.2017;8: 1

42. Ahmed B, Kinfe K, Ayisha A. Prevalence study of sheep lice and species composition in Tiyo district of Arsi zone. Journal of Biology, Agriculture and Healthcare. 2017; 7(3): ISSN 2224-3208.

43. Jemere B, Marta T, Rahmeto A. External parasite infestation in small ruminants in Wolmera District of Oromia Region, Central Ethiopia. Journal of Animal and Veterinary advances.2011; 4: 518-523.

44. Asnake F, Yacob H, Hagos A.Ectoparasites of small ruminants in three agro-ecological districts of southern Ethiopia. African Journal of Basic \& Applied Sciences, 2013; 5 (1): 47-54.

45. Sisay A, Yilkal A, Yacob H. Ectoparasites of Sheep and Goats in North-West Amhara Regional State, Ethiopia. EthiopianVeterinary Journal.2013;17 (1): 55-67

46. Enquebaher K, Etsay K. Epidemiological study on manage mite, lice and sheep keds of small ruminants in Tigray region, northern Ethiopia. Ethiopian Veterinary Journal.2010;14: 51-65 
47. Aziz FB,Awal MA, Hasan Q, Rahman MM. Comparative efficacy of Ivermectin and Diazinon against ectoparasites in sheep. Journal of Animal and Veterinary Advances, 2004; 37: 398-46.

48. Comparative efficacy of Ivermectin and Diazinon against ectoparasites in sheep. Food and Agricultural organization of United Nations. (http://www.doaj.org; accessed online on 5/03/2019).2012.

49. Hanif MA, Mostofa M, Choudhury EM, Awal AM, Rahman MM. Efficacy of ivermectin (pour on formulation) against ectoparasites in sheep. Bangladesh Journal of Veterinary Medicine.2005; 3:140-143.

50. Hassan MM, Ahasanul MD, Hoque MA, Islam SK,Khan SA. Efficacy of anthelmintic against parasitic infections and their treatment effect on the production and blood indices in Black Bengal goats in Bangladesh. Turkish Journal of Veterinary Science, 2012; 36(4): 400-408.

51. Byaruhanga C, Okwee-Acai J. Efficacy of albendazole, levamisole and ivermectin against gastrointestinal nematodes in naturally infected goats at the national semi-arid resources. Research Institute, Serere, Uganda. Veterinary Parasitology. 2013; 195: 183-186.

52. Tsotetsi AM, Njiro S, Katsande TC, Mayo G, Baloyi F,Mpofu J. Prevalence of gastrointestinal helminths and anthelmintic resistance on small-scale farms in Gauteng Province, South Africa. Tropical Animal Health and Productio 2013;45: 751-761.

\section{Figures}




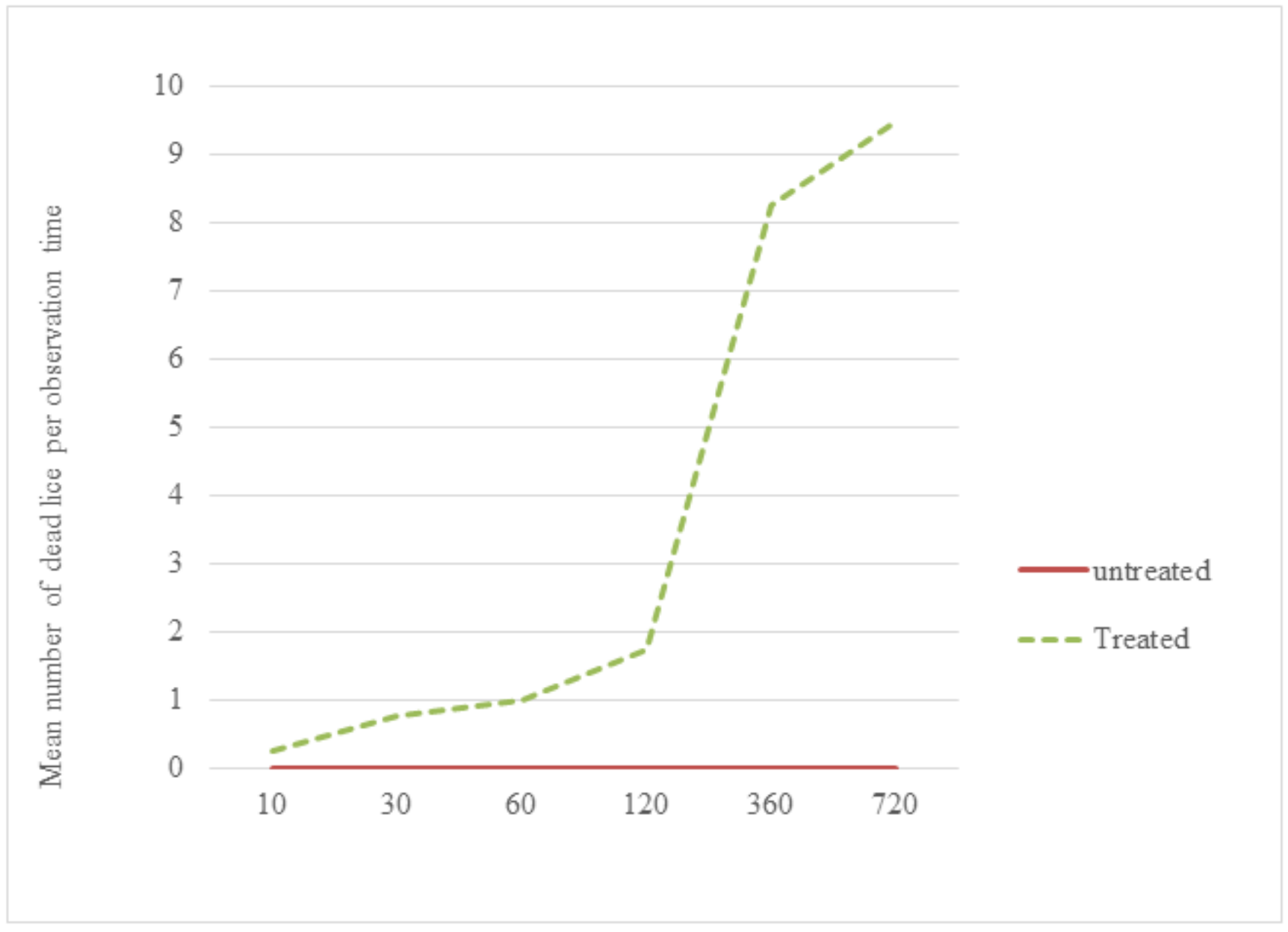

Figure 1

In-vitro mean dead lice count after $60 \%$ diazinon treatment in sayint district 


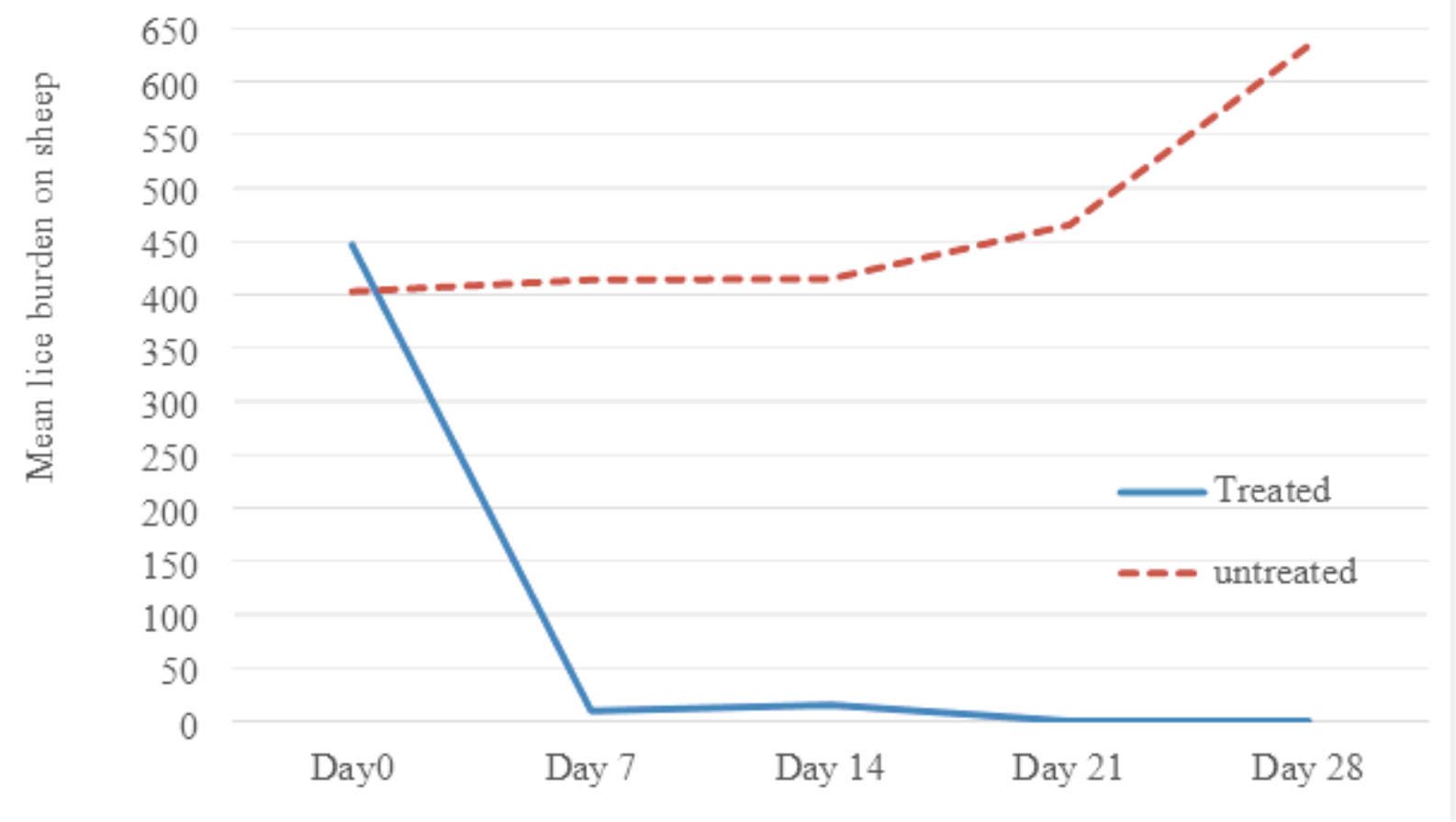

Figure 2

In-vivo mean lice burden of sheep treated with $60 \%$ Diazinon per different days of inspection

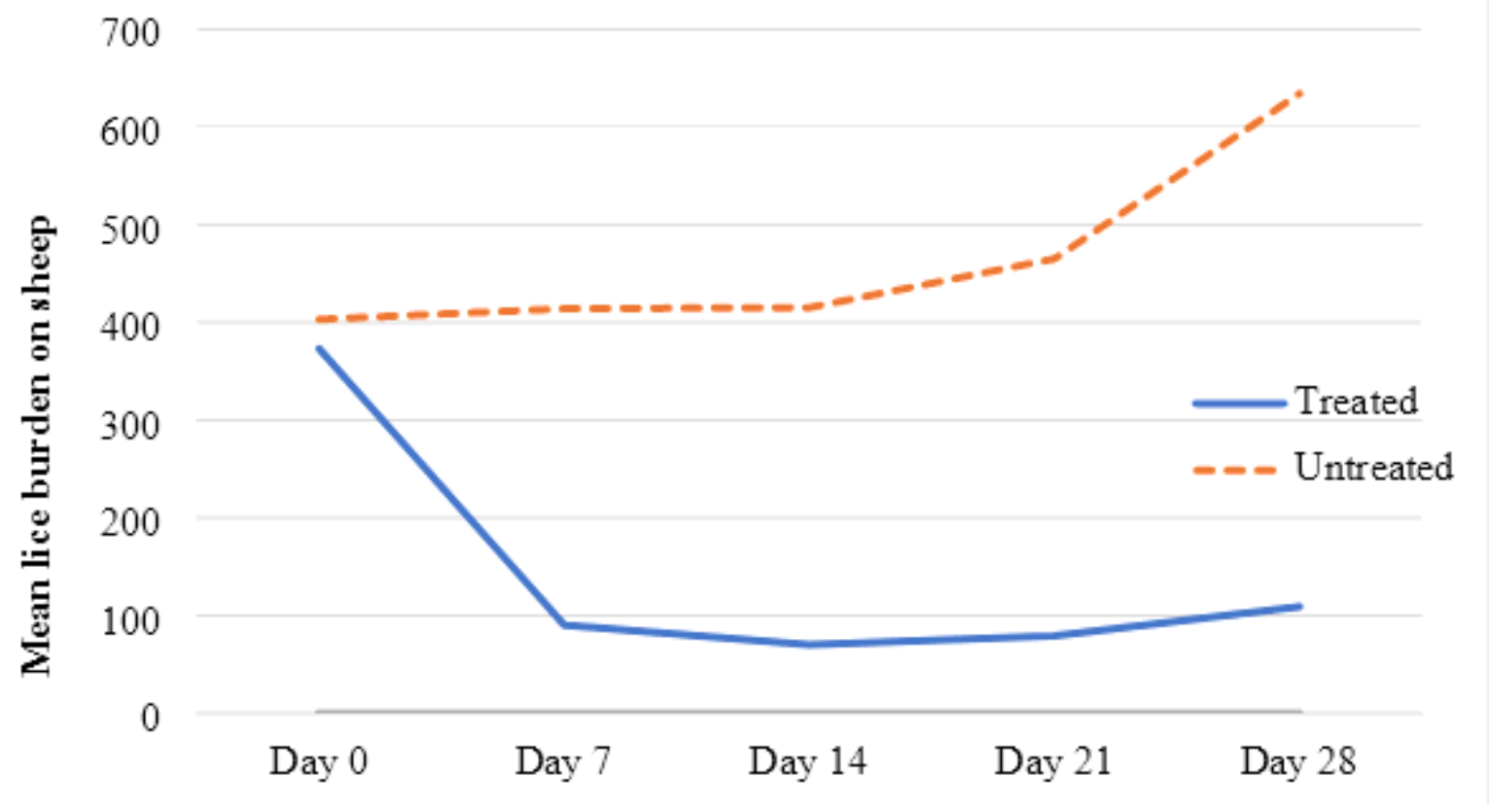

Figure 3 
In vivo mean lice burden for $1 \%$ ivermectin per days of inspection in sayint district

\section{Supplementary Files}

This is a list of supplementary files associated with this preprint. Click to download.

- supplement5.pdf 